\title{
Vasoactive Agents Do Not Adversely Influence the Success of Maxillo-Facial Microvascular Free-flap Surgery: A Retrospective Analysis
}

Research Article

\author{
L Rose $^{1 *}$, N Prado ${ }^{1}$, D Mulvey ${ }^{2}$, D Laugharne ${ }^{1}$, K Jones $^{1}$, J Stenhouse
}

${ }^{1}$ Department of Oral \& Maxillo-Facial Surgery, Derby Teaching Hospitals NHS Trust, Royal Derby Hospital, Uttoxeter Road, Derby, UK.

${ }^{2}$ Department of Anaesthesia \& Critical Care, Derby Teaching Hospitals NHS Trust, Royal Derby Hospital, Uttoxeter Road, Derby, UK.

\section{Abstract}

Introduction: Microvascular free-flap surgery has become the gold standard in Head and Neck oncology reconstruction with published success rates of $\geq 95 \%$. Evidence suggests that poor blood flow to the flap remains the primary cause of failure and this might be linked to the type of vasoconstrictor used during anaesthesia.

Aims: The objectives of this analysis were (1) an assessment of the success rate of microsurgical free-flaps placed by the Oral and Maxillo-Facial surgeons at the Royal Derby Hospital, (2) whether the intraoperative administration of vasoactive agents had a negative effect on this success, (3) to investigate whether type and/or method of administration of vasoactive agents influences flap success rates.

Methods: A sample of 123 consecutive patients undergoing surgery in an eight-year period were analysed retrospectively. Data collected included the type of free-flap used, the success of the procedure, the type of vasoconstrictor used and the drug administration method.

Results: There were 119 successful flaps and 4 failures giving a success rate of $97 \%$ in the study period. Two vasoconstrictors (noradrenaline and metaraminol) were used intraoperatively, either alone or in combination. These agents were administered by differing methods depending on anaesthetic choice. No association was found between the use of vasoconstrictors and flap failure. Nor was there an association between the method of vasoconstrictor administration and flap failure.

Conclusions: This analysis of a limited number of patients indicates that administration of a vasoconstrictor appears to have no detrimental effect on the success rate of microvascular free-flap surgery. Further, the type of vasoconstrictor and mode of administration does not appear to influence these success rates.

\section{Background}

Microsurgical free-flaps were first described in 1964 [1] and have become the gold standard reconstructive technique in head \& neck surgery following oral cancer resection [2]. Rapid developments in methodology have allowed progression from pedicled flaps to microsurgical free-flaps in a relatively short space of time [3]. Five main types of free-flap are used in the majority of oncology cases. These include; radial forearm, fibula, rectus abdominis, jejunum and antero-lateral thigh flaps [4]. Radial forearm free-flaps are favoured at Royal Derby Hospital due to their versatility and reliability in the transfer of well vascularised tissue. This procedure was first published in 1981 by Yang et al., [5] and it is now the leading microsurgical free-flap used for head \& neck reconstruction [6].
Currently there are no national standards against which a local success rate for free tissue transfer surgery can be measured. However published data suggest success rates in excess of $95 \%$ are typical for an expert centre [4-12]. For the 5\% of microvascular free-flaps that fail it is suggested that venous thrombosis remains the primary cause, often within 48 hours of surgery [7]. Numerous possible explanations for this morbidity exist including human error, technical failures, and geometrical positioning [7]. There is a body of opinion which suggests that anaesthetic drugs used intraoperatively may be an important factor in failure [13].

Concern centres on the use of vasoconstrictor agents to maintain haemodynamic stability as these agents could affect regional blood flow to the flap [13] and consequently there is inconsistent use of such drugs in published case series. In a recent survey by Motakef et al., 2015 [14], 46\% of respondents considered the use

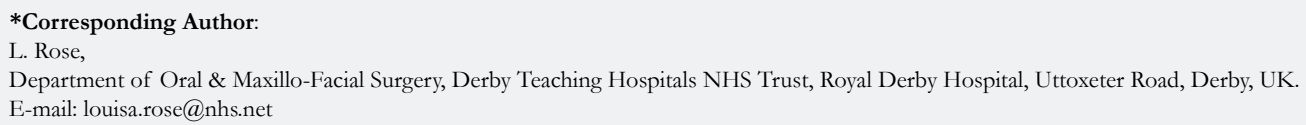

Copyright: $\mathbf{L}$ Rose $^{\circ}$ 2016. This is an open-access article distributed under the terms of the Creative Commons Attribution License, which permits unrestricted use, distribution and reproduction in any medium, provided the original author and source are credited. 
of noradrenaline to be contraindicated in microsurgical free-flaps. However, vasoconstrictors including noradrenaline have been cited as being of benefit for maintaining flap blood flow. Several authors state that use of vasoactive agents causes no increase in failure or complication rates during head \& neck free-flap surgery $[15,16]$.

Non-pharmacological factors such as surgical and anaesthetic technique can vary between institutions and individual practitioners and may influence success rates. At the Royal Derby Hospital, head \& neck free-flap surgery is undertaken by 3 maxillofacial surgeons and 4 anaesthetists limiting this variability to a large extent and allowing focus to be centred on vasoconstrictor usage.

\section{Definitions}

Success: A flap is considered to be a success if there is no postoperative requirement for replacement of the transferred tissue or post-operative flap necrosis.

Salvage: Flaps that required a secondary procedure (such as reanastomosis of the microvasculature) but subsequently remained viable are considered successful for the purpose of this analysis.

Failure: A flap is considered a failure if it becomes necrotic and requires removal and/or replacement from a new donor site within 14 days post-surgery.

\section{Aims}

The aims of this analysis are as follows:

- To determine the current success rate of microvascular freeflap surgery in the Maxillo-Facial department at RDH.

- To determine whether the use of vasoactive agents has any effect on the success rates of microvascular free-flaps.

- To investigate whether type and/or method of administration of vasoactive agents influences flap success rates.

\section{Methods}

Data was collected retrospectively from a $\log$ of consecutive patients undergoing microvascular free-flap surgery from September 2007 to September 2015. This time frame was chosen to ensure consistency of the performing consultants within the department.

A standard sample size calculator was used to provide an adequate group size for the study. The assumption for the size was based on estimation from theatre log-books by experienced consultants operating during a given calendar period. It was estimated that around 150 patients had been treated with microvascular free-flap surgery during the allotted time frame. Based on this number and using a $95 \%$ confidence level with a confidence interval of 5 , a group size of 108 was calculated [17].

The eligible patients were confirmed by examining the Operating Theatre logbooks from the Theatres used for the relevant surgeries (Only 2 specific Operating Theatres are used within the hospital. All logbooks were identified and complete with no gaps in timeline) and cross referenced against the coding logs for the relevant procedures.

126 patients were identified in total. As this number was not far from the calculated sample size of 108 , we decided to study all 126.

\section{Inclusion Criteria}

- Any patient who underwent microvascular free-flap surgery operated on by consultants in the Head \& Neck department at Royal Derby Hospital, between the dates of $1^{\text {st }}$ September 2007 to $1^{\text {st }}$ September 2015.

\section{Exclusion Criteria}

- Illegible records (anaesthetic or clinical).

- $\quad$ Pedicled flaps (as not true free-flaps).

2 sets of patient notes were found to have illegible anaesthetic records. Therefore the vasoactive agent administration could not be assessed appropriately and so these patients were removed from the analysis.

1 patient was identified to be coded as having a microvascular free-flap however, clinical notes confirmed the patient had undergone a Pec Major, pedicled flap. This patient was removed from the analysis.

Final sample size: 123

Data was collected by two of the authors involved in this analysis (L. Rose and N. Prado). Patients were identified as outlined above and the clinical notes were requested (including anaesthetic notes). A data collection e-form was designed and analysed using Microsoft Excel. The data collection included the following;

- Hospital number.

- Flap type.

- Success of flap (success/salvage/failure).

- Vasoconstrictor used: noradrenaline/metaraminol.

- Administration by single bolus, multiple boluses or infusion

Each data collector initially analysed all patient notes separately and the data was then secondly confirmed together to ensure accuracy, reliability and consistency.

\section{Ethical Approval}

An online Medical Research Council questionnaire provided by the Health Research Authority indicates that this study does not need NHS Research Ethics Committee approval. The link to this source has been provided within the references [18].

The reasons for this are as follows:

- The study was not initially intended to be research and therefore patients were not recruited as such. The patients identified retrospectively had their treatment based on clinical symptoms and need, not based on research outcomes.

- The data analysed is data that is routinely collected for these types of patients as per protocol in Head \& Neck oncology surgery at Royal Derby Hospital. 
- All data is anonymous with no breach of confidentiality.

\section{Results}

During the analysis period 6 types of micro vascular free-flap were used and the distribution is shown in Figure 1.

The analysis showed that 2 vasoactive agents were used; noradrenaline and metaraminol, as well as a combination of the two (Figure 2). These were given via a variety of methods; continuous infusion, multiple bolus or single bolus (Figure 3).

Of 123 free-flap cases analysed, 119 (97\%) were successful. Within this sub-set of 119 successful cases, 90 received a vasoconstrictor (noradrenaline and metaraminol) either alone or in combination. 48 of these 90 successful cases received a continuous infusion of drug throughout surgery and 35 of the 90 received multiple boluses. A minority of 7 cases received a single bolus of vasoconstrictor

11 out of the 119 successful cases $(9 \%)$ required a salvage procedure to sustain viability and in 10 cases an exploration and/ or microvasculature re-anastamosis was required. The remaining salvage case underwent superficial layer removal only, as the main body of the flap was viable. During initial surgery of these salvage procedures, 8 of the 11 patients received a vasoconstrictor (2 noradrenaline and 6 metaraminol) by a variety of administration methods ( 3 infusion, 4 multiple bolus and 1 single bolus).

4 flaps failed; 2 patients had undergone a radial forearm flap procedure, 1 a fibula flap and 1 a rectus abdominus flap. 3 of these failed flap cases had received a vasoconstrictor; 1 metaraminol infusion, 1 noradrenaline infusion and 1 patient received a single bolus of both noradrenaline and metaraminol.

\section{Discussion}

These results show a 97\% success rate for microvascular free flap surgery at Royal Derby Hospital, mirroring the success rate of $\geq 95 \%$ widely reported in the international literature $[4,7,9,10$, $11,12]$.

The data above suggests that the intraoperative administration of vasoactive agents does not have a consistently detrimental effect on the success rate of free-flap surgery.

Although 3 of 4 failed cases in this analysis were given a vasoconstrictor, 90 of the 119 successful procedures (76\%) also received such an agent. The other 29 successful procedures $(24 \%)$ and 1 failed case did not receive a vasoconstrictor. The ratios of failures in each group are similar (vasoconstrictor $3 / 93(3.2 \%)$ vs non-constrictor $1 / 30(3.3 \%))$.

10 cases underwent salvage surgery due to microvascular failure. These results do not support the premise that this resulted solely from the use of vasoconstrictors during the primary operation.

The method chosen for vasoconstrictor administration was dependent upon anaesthetic preference, and 48 of the 119 successful cases had a vasoconstrictor administered via continuous infusion throughout surgery. Given the protracted duration of free-flap surgery it cannot be argued that sustained plasma levels of vasoconstrictor have a negative influence on free-flap success rate.

Figure 1. Types of free-flaps used, DCIA = deep circumflex iliac artery, ALT = antero-lateral thigh.

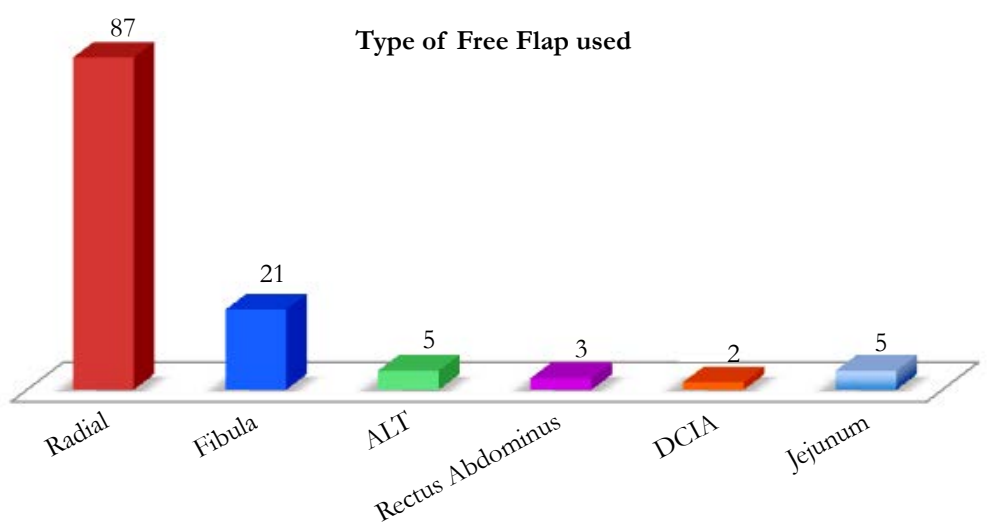

Figure 2. Types of vasoconstrictor used during free-flap surgery.
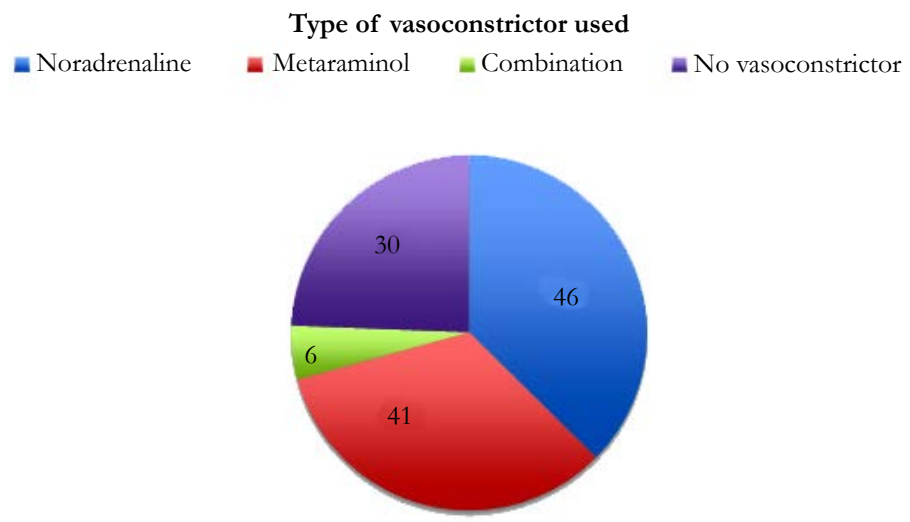
Figure 3. Method of vasoconstrictor administration during free-flap surgery.

a Single Bolus aultiple Bolus $\quad$ Infusion

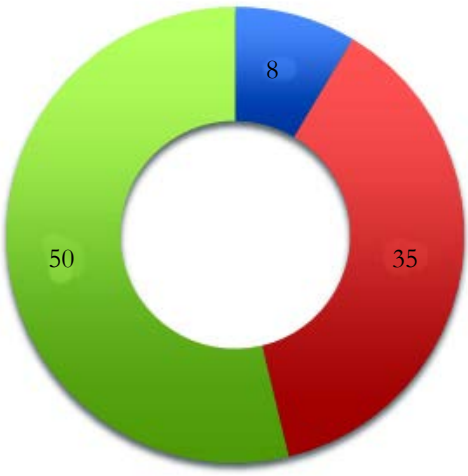

However a contrary view to these conclusions has been expressed in the literature $[14,15,19,20]$.

\section{Limitations}

The authors recognise that this analysis has a number of flaws. Only a small number of patients are included and together with the low failure rate it is difficult to make a definitive statistical conclusion about the effect of vasoconstrictors on flap success. However the data does suggest that there appears to be no consistent detrimental effect to their usage, which is contrary to the usual expert opinion.

It must also be noted that due to the small patient group, there were patients who received or did not receive one or two drugs, single or multiple times. Such an approach makes it difficult to assess the therapy-effect relationship and the specific role of sympathomimetics in flap failure rates.

However, we hope that this retrospective pilot analysis will pave the way for future prospective trials, with appropriate statistical design to answer the important question of whether intraoperative vasoconstrictors are friend or foe to microvascular free flaps.

\section{Conclusions}

Although this analysis involves a limited number of patients it may suggest that administration of vasoconstrictors appears to have no detrimental effect on the success rate of microvascular free-flap surgery. Further, the mode and type of vasoconstrictor administered does not appear to influence these success rates.

\section{References}

[1]. Nakayana K, Yamamoto K, Tamiya T, Makino H, Odaka M, Ohwada M and Takahashi H (1964) Experience with free autografts of the bowel with a new venous anastomosis apparatus.Surgery. 55: 796-802.

[2]. Lutz BS and Weiz FC (2005) Microsurgical workhorse flaps in head and neck reconstruction. Clin plastic surg. 32(2): 421-430.

[3]. Rinku KG and Krishnamurthy A (2013) Microsurgical free flaps: Controversies in maxillofacial reconstruction. Ann Maxillofac surg. 3(1): 72-79.

[4]. Disa JJ, Pusic AL, Hidalgo DH and Cordeiro PG (2001) Simplifying microvascular head and neck reconstruction: a rational approach to donor site selection. Ann plastic surg. 47(4): 385-389.

[5]. Yang G, Chen B and Gao Y (1981) Forearm free skin flap transplantation. Natl Med J China. 61: 139- 41.
[6]. Futran ND, Gal TJ and Farwell DG (2003) Radial forearm free flap. Oral and Maxillofacial surgclin am. 15(4): 577-591.

[7]. Novakovic D, Patel RS, Goldstein DP and Gullane PJ (2009) Salvage of failed free flaps used in head and neck reconstruction. Head and neck oncology. 21(1): 33 .

[8]. Suh JD, Sercarz JA, Abemayor E, Calcaterra TC, Rawnsley JD, Alam D and Blackman KE (2004) Analysis of outcome and complications in 400 cases of microvascular head and neck reconstruction. Arch otolaryngol head neck surg. 130(8): 962-6.

[9]. Blackwell KE, Brown MT and Gonzalez D (1997) Overcoming the learning curve in microvascular head and neck reconstruction. Arch otolaryngol head neck surg. 123(12): 1332-5.

[10]. Cordeiro PG and Hidalgo DA (1995) Conceptual considerations in mandibular reconstruction. Clinplassurg. 22(1): 61-9.

[11]. Khouri RK and Shaw WW (1989) Reconstruction of the lower extremity with microvascular free flap: a 10 -year experience with 304 consecutive cases. J Trauma. 29(8): 1086-94.

[12]. Bianci R, Capelli C, Ferrari S, Feri A and Sesenna E (2009) Free flaps outcomes and complications in head and neck reconstruction. J Craniomaxillofac Surg. 37(8): 438-442.

[13]. Pereira C, Figueiredo M, Carvalho R, Catre D and Assunção J (2012) Anesthesia and surgical microvascular flaps. Rev. Bras. Anestesiol. 62(4): 563-79.

[14]. Motakef S, Mountziaris PM, Ismail IK, Agag RL and Patel A (2015) Perioperative management for microsurgical free tissue transfer: survey of current practices with a comparison to the literature. J Reconstr Microsurg. 3(5): 355-63.

[15]. Monroe MM, Cannady SB, Ghanem TA, Swide CE and Wax MK (2011) Safety of vasopressor use in head and neck microvascular reconstruction: a prospective observational study. Otolaryngol Head Neck Surg. 144(6): 877-882.

[16]. Chen C, Nguyen MD, Bar-Meir E, Hess PA, Lin S, Tobias AM, Upton J and Lee BT (2010) Effects of vasopressor administration on the outcomes of microsurgical breast reconstruction. Ann PlastSurg. 65(1): 28-31.

[17]. Creative Research Systems, The Survey System [online], Accessed 29/08/2016. <http://www.surveysystem.com/sscalc.htm>.

[18]. NHS Health Research Authority MRC, Medical Research Council [online], Accessed 29/08/2016. <http://www.hra-decisiontools.org.uk/ethics/ notresearch.html>.

[19]. Monroe MM, McClelland J, Swide C and Wax MK (2010) Vasopressor use in free tissue transfer surgery. Otolaryngol Head Neck Surg. 142(2): $169-173$.

[20]. Eley K, Young D and Watt-Smith S (2012) Epinephrine, Norepinephrine, Dobutamine, and Dopexamine Effects on Free Flap Skin Blood Flow. Plastic Reconstructive Surgery. 130(3): 564-70. 